Supporting Information 


\section{Graphene Quantum Dots Sensitized ZnO-Nanorods/GaN- Nanotowers Heterostructure based High Performance UV Photodetector}

Lalit Goswami ${ }^{1,2}$, Neha Aggarwal, Rajni Verma ${ }^{3}$, Swati Bishnoi ${ }^{1}$, Sudhir Husale $^{1}$, Rajeshwari Pandey ${ }^{2}$ and Govind Gupta ${ }^{1, \dagger, *}$

${ }^{1}$ CSIR-National Physical Laboratory, Dr K.S. Krishnan Road, New Delhi- 110012, India.

${ }^{2}$ Department of Electronics \& Communication Engineering, Delhi Technological University, New Delhi110042.

${ }^{3}$ The University of Melbourne, Parkville, Victoria 3010, Australia.

'Academy of Scientific \& Innovative Research, CSIR-HRDC Campus, Ghaziabad, Uttar Pradesh - 201002, India.

*Corresponding Author: govind.npl@nic.in; Telephone: +91-1145609503

\section{$\underline{\text { Supporting Information }}$}

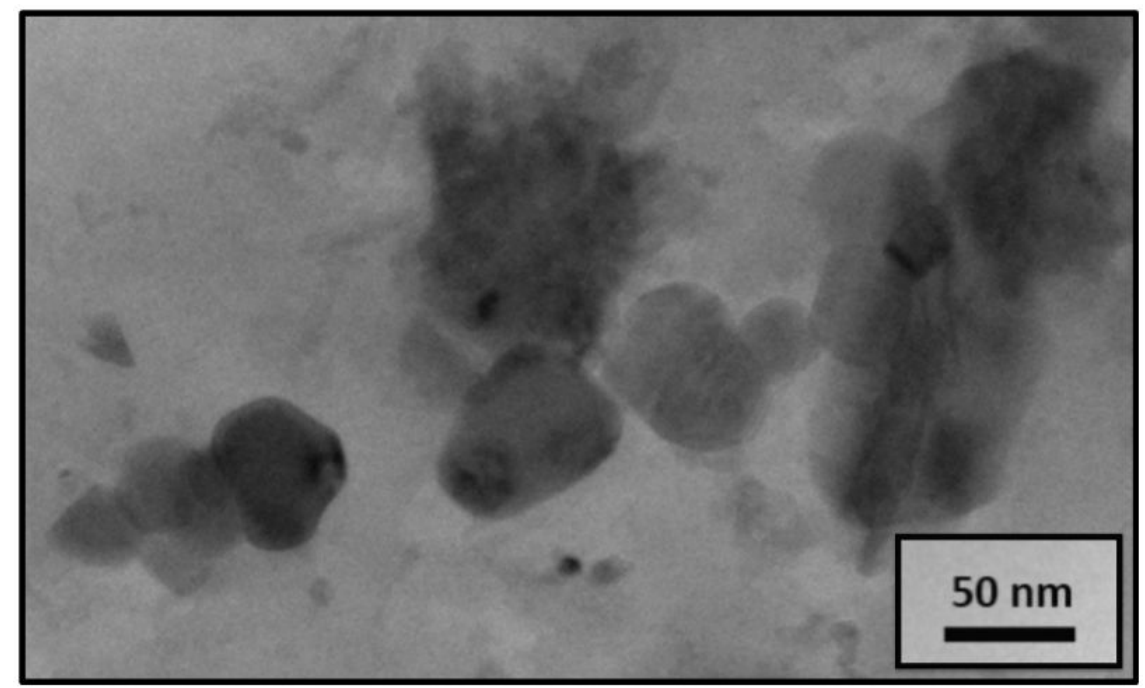

Figure S1. HRTEM image of chemically synthesised GQDs. 


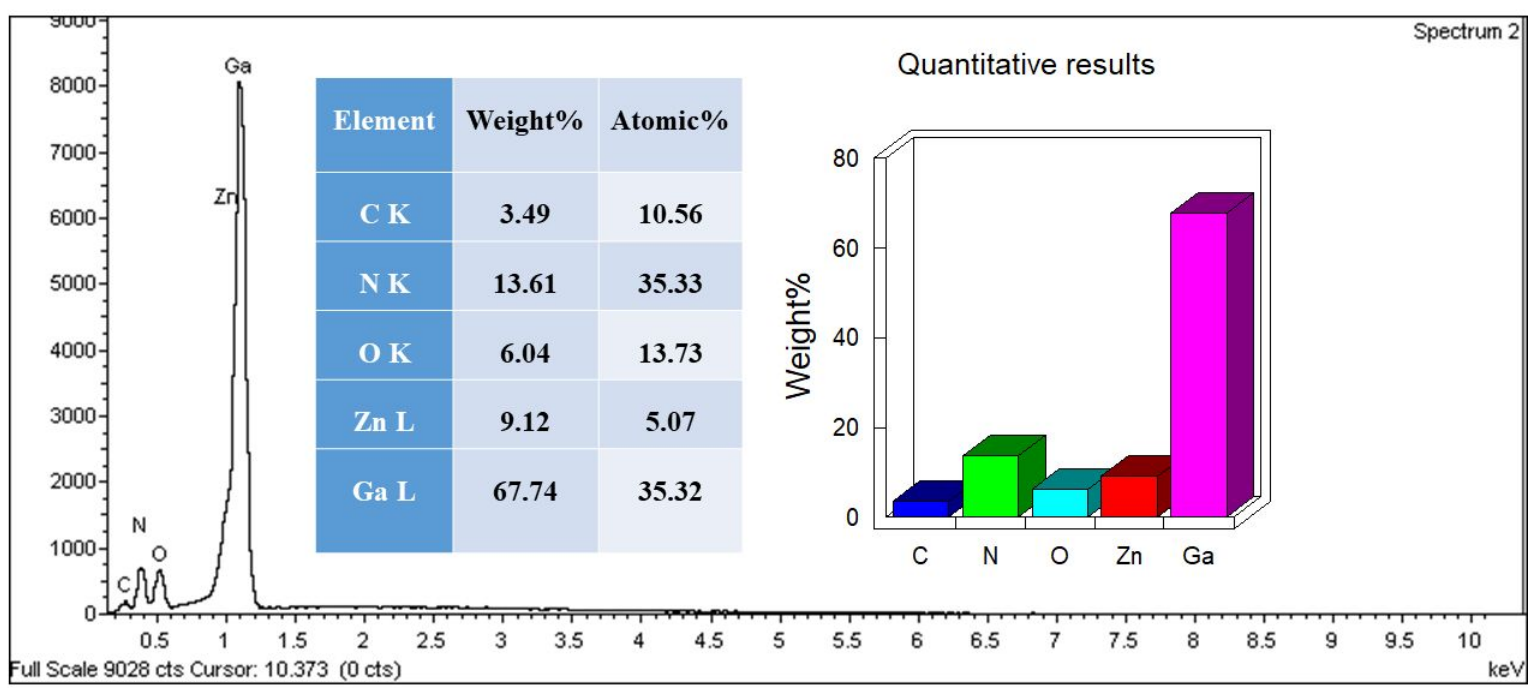

Figure S2. EDS spectra of as grown GQDs--ZnO/GaN heterostructure.
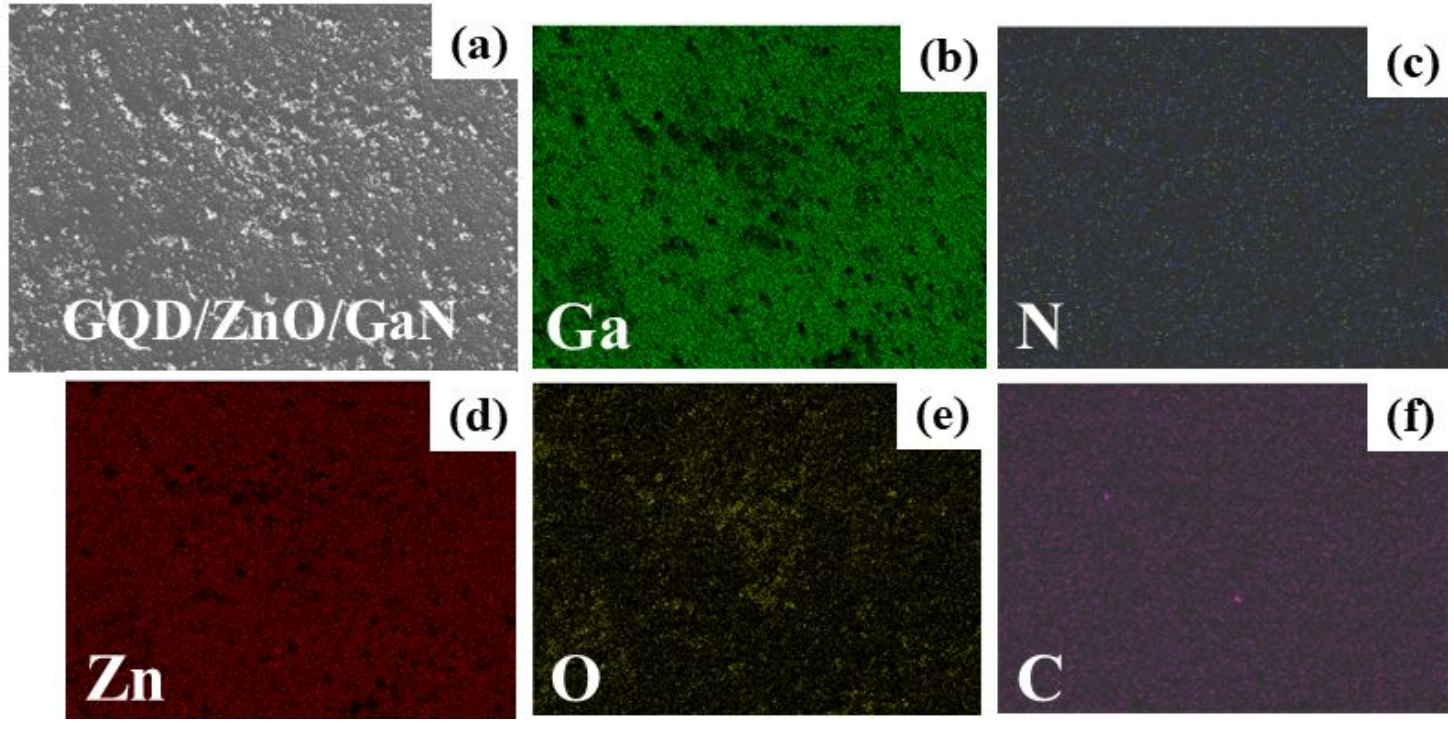

Figure S3. Uniform distribution of various element involved in GQDs--ZnO/GaN heterostructure by elemental mapping technique (a) Top view FESEM image, (b) Gallium (Ga), (c) Nitrogen (N), (d) Zinc (Zn), (e) Oxygen (O) and (f) Carbon (C) stand for GQDs presence. 

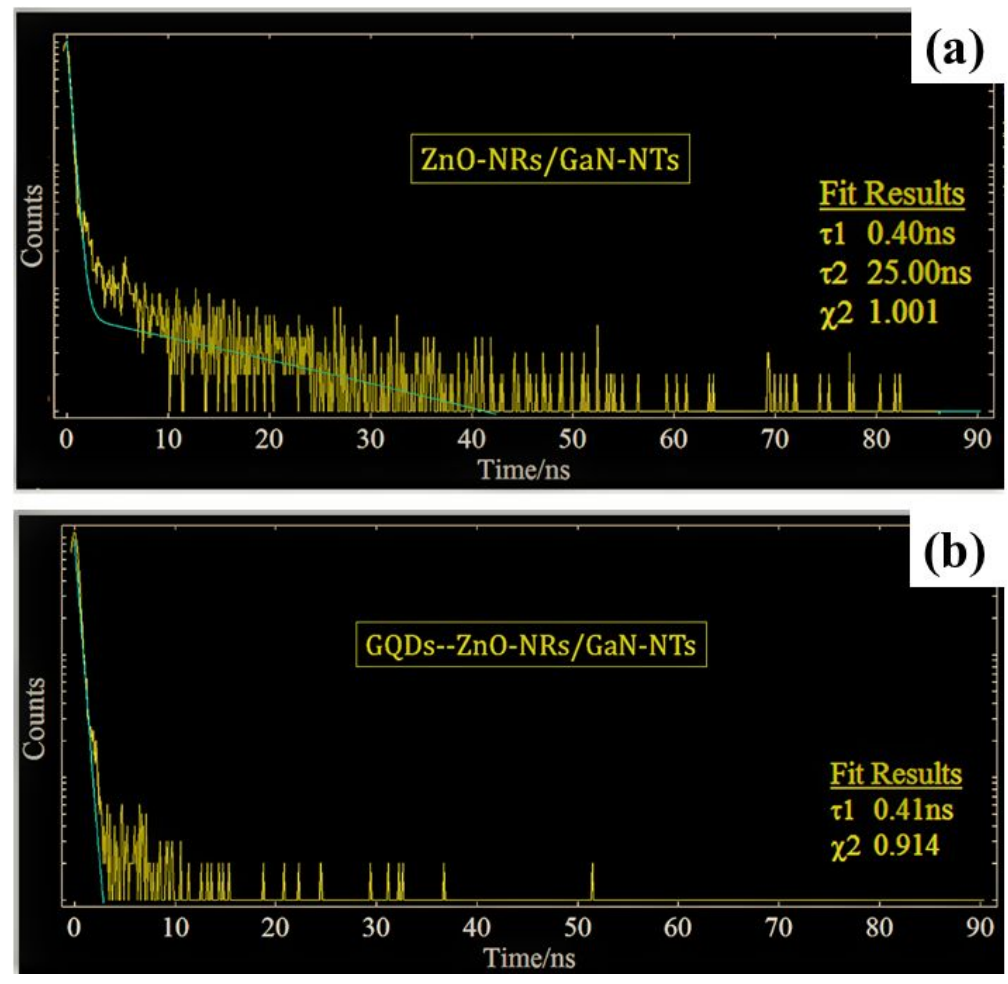

Figure S4. Time resolve photoluminescence spectra of (a) bare ZnO-NRs/GaN-NTs and (b) GQDs-ZnO-NRs/GaN-NTs heterostructures.

The raw \& fitted TRPL data of bare \& GQD functionalized ZnO-NR/GaN-NT films. Sluggish waved signals represent the raw (experimental) data while the solid lines are showing fitted data using the following two-order exponential decay formula:

$$
y=y_{0}+y_{1} \exp \left(-\frac{t}{\tau 1}\right)+y_{2} \exp \left(-\frac{t}{\tau 2}\right)
$$

where $\mathrm{y}$ is the emission intensity, $\mathrm{y} 0, \mathrm{y} 1, \mathrm{y} 2$ are constant, $\mathrm{t}$ is time.

There are multiple parallel current conduction channels offered by multiple interfaces $(\mathrm{ZnO} / \mathrm{GaN}$, GQDs/GaN and GQDs/ZnO/GaN) were available to summarised the photocurrent of both the devices such as $I_{A}$ and $I_{B}$. The flow of current in the device can be well understood, wherein, the bare $\mathrm{ZnO} / \mathrm{GaN}$ heterojunction based UV photodetector's photocurrent $\left(\mathrm{I}_{\mathrm{A}}\right)$ found to be lesser as compared to GQDs sensitization injected extra charge carrier enhanced photocurrent $\left(\mathrm{I}_{\mathrm{B}}\right)$ as shown in figure $\mathrm{S} 5$. 
(a)

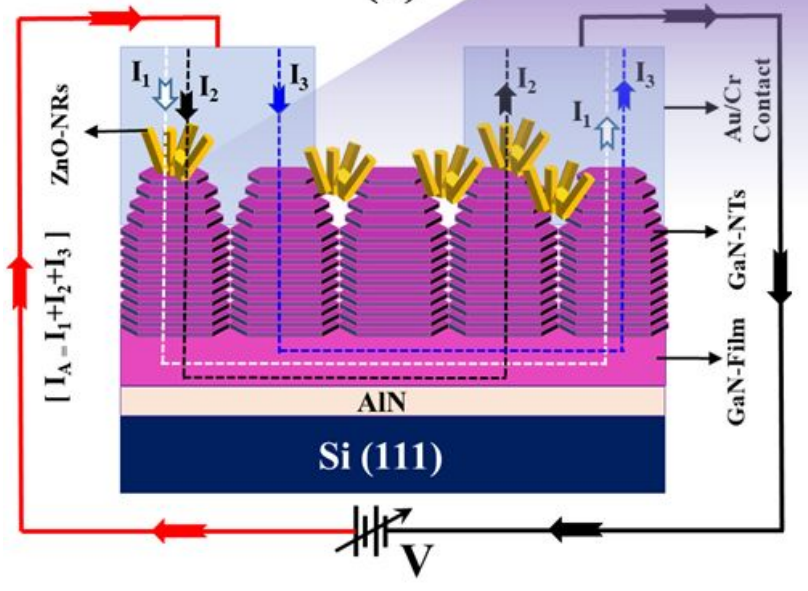

(b)

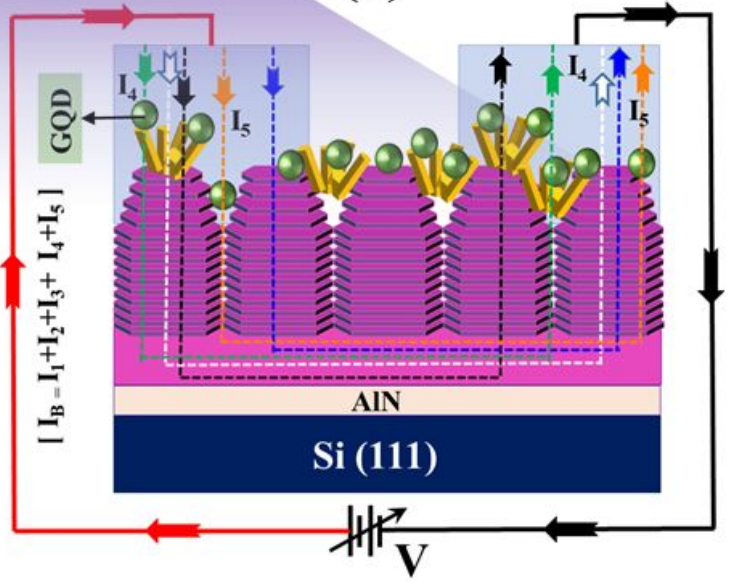

Figure S5. Schematic diagram of opto-electrical transport taken place in fabricated (a) bare ZnO/GaN and (b) GQDs sensitized UV photodetectors.

Moreover, for better understanding, other schematic (figure S6) has been visualized to represent the circuitry involved to deliver the photocurrent. Thereby, parallel and series combination of optically sensitive resistance offered by the various semiconductors and their heterojunctions is represented in best possible way.

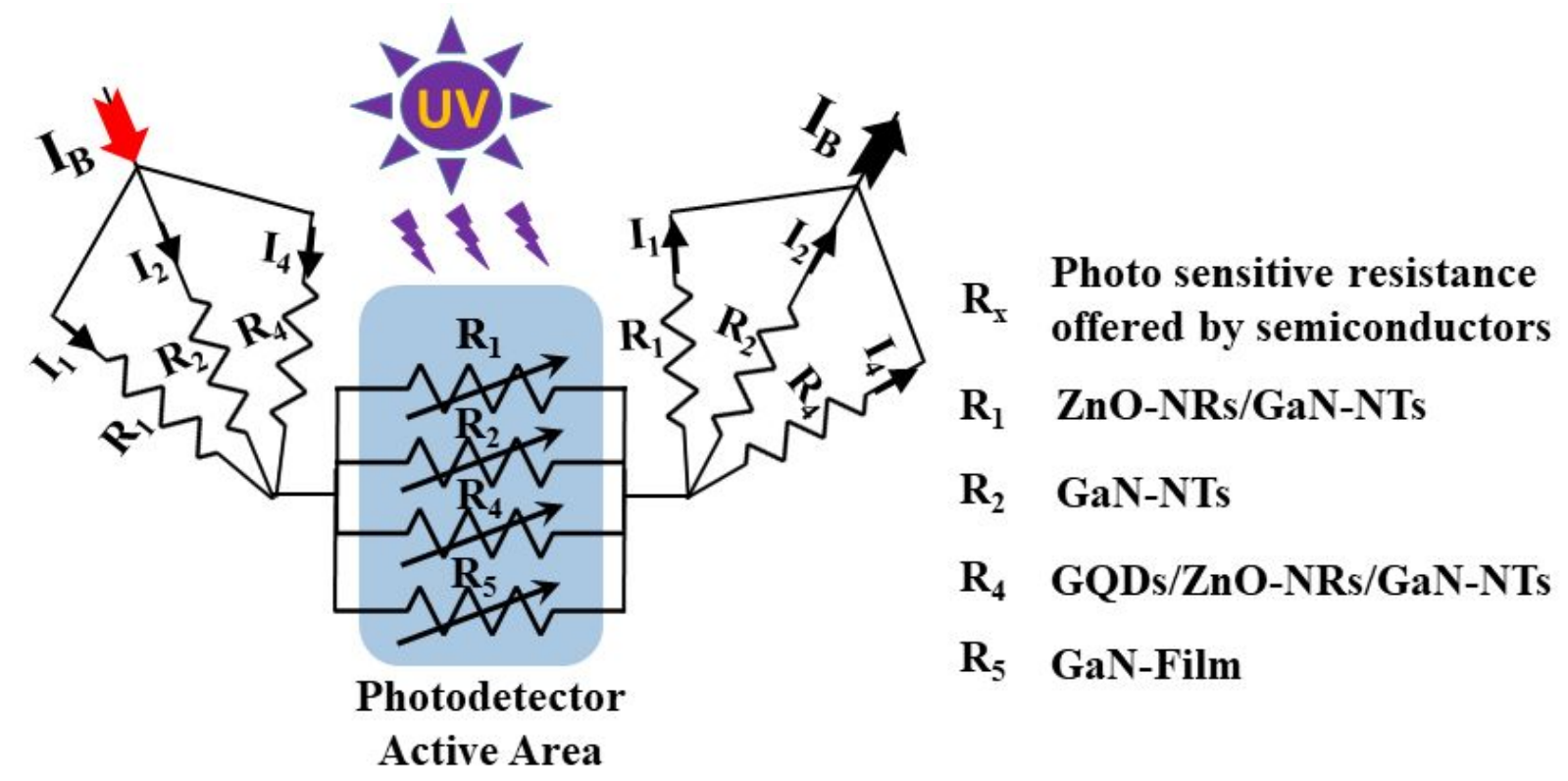

Figure S6. Schematics of circuitry involved to deliver the photocurrent. 